\title{
Relationship between White Matter Hyperintensities and Hematoma Volume in Patients with Intracerebral Hematoma
}

\author{
Xuemei Chen ${ }^{1,3,4}$, Yuexinzi Jin ${ }^{1}$, Jian Chen ${ }^{1}$, Xin Chen ${ }^{1}$, Xiang Cao ${ }^{2,3,4}$, Linjie Yu ${ }^{2}$, Yun \\ $\mathrm{Xu}^{1,2,3,4, *}$
}

\begin{abstract}
${ }^{1}$ Department of Neurology, Nanjing Drum Tower Hospital Clinical College of Nanjing Medical University, Nanjing 210008, China

${ }^{2}$ Department of Neurology, Affiliated Drum Tower Hospital, and Jiangsu Key Laboratory for Molecular Medicine, Nanjing University Medical School, Nanjing 210008, China.

${ }^{3}$ Jiangsu Province Stroke Center for Diagnosis and Therapy, Nanjing 210008, China

${ }^{4}$ Nanjing Neuropsychiatry Clinic Medical Center, Nanjing 210008, China
\end{abstract}

[Received October 27, 2017; Revised December 20, 2017; Accepted January 8, 2018]

\begin{abstract}
The relationship of white-matter hyperintensity (WMH) to intracerebral hemorrhage (ICH) remains unclear. In this retrospective study, we investigated whether the severity and progression of WMH could be related to the hematoma volume and absorption in ICH. 2338 WMH patients with ICH aged $\geq 40$ years receiving brain computed tomography (CT) imaging within 12 hours of ICH symptom onset were screened, and 227 patients were included in the final study. The severity and progression of WMH were assessed using the software programs MRICRON and ITK-SNAP on brain magnetic resonance imaging (MRI) and the hematoma volumes and absorption with ITK-SNAP software on CT. We assessed the association of WMH severity with ICH volume in 227 patients at baseline. Totally 183 of 227 patients underwent repeated CT within 14 days of ICH onset. The relationship of WMH severity to ICH absorption was analyzed in 183 patients. Additionally, among all 227 patients, 37 subjected to another MRI before ICH onset were divided into two groups according to WMH progression: non-progression and progression groups. The link between WMH progression and hematoma volume was examined. The ICH volume was significantly larger in patients with the highest WMH scores than in those with the lowest WMH scores. Larger WMH volume was independently associated with larger ICH volume (odds ratio 1.00; 95\% CI, 1.00 to $1.00 ; P=0.049$ ). There was a trend towards WMH progression being related to ICH volume $(P=0.049)$. Contrastingly, the WMH volume was not linked with hematoma absorption $(P=\mathbf{0 . 7 9})$. In conclusion, we found that greater severity and progression of WMH were associated with larger ICH volume. Our findings suggest that WMH might provide important prognostic information about patients with ICH and may have implications for treatment stratification.
\end{abstract}

Key words: intracerebral hemorrhage, white matter hyperintensities, hematoma, leukoaraiosis, quantitative analysis

Intracerebral hemorrhage (ICH) is usually caused by the rupture of small penetrating arteries secondary to hypertensive changes or other vascular abnormalities [12 ]. ICH accounts for approximately $10-20 \%$ of all stroke cases and is associated with high morbidity and mortality [3-4]. Common factors influencing ICH include hypertension, cigarette smoking, excessive alcohol consumption, decreased low-density lipoprotein (LDL)

*Correspondence should be addressed to: Dr. Yun Xu, Department of Neurology, Nanjing Drum Tower Hospital Clinical College of Nanjing Medical University, Nanjing 210008, China. Email: xuyun20042001@aliyun.com

Copyright: () 2018 Chen X et al. This is an open-access article distributed under the terms of the Creative Commons Attribution License, which permits unrestricted use, distribution, and reproduction in any medium, provided the original author and source are credited. 
cholesterol levels, decreased triglyceride (TG) levels and usage of certain drugs [5]. The spontaneous intracerebral hemorrhage results from small vessel disease (SVD) [6]. There are some MRI markers for SVD, like white matter hyperintensity (WMH), lacunar infarctions (LIs), cerebral microbleeds (CMBs) and perivascular spaces (PVS) [7]. $\mathrm{WMH}$, or leukoaraiosis, represents areas of ischemic white matter damage attributed to degenerative changes to small vessels, including intimal hyperplasia, atherosclerosis, lipohyalinosis and amyloidosis [8]. Therefore, WMH might reflect the vulnerability of individual brains to pathologic insults [9]. Most researches focus on WMH is associated with ischemic stroke and less on ICH. Our previous research shown that $\mathrm{WMH}$ progression within the first two years was an independent predictor of LIs development. One previous publication also indicated greater WMH burden was associated with small vessel stroke compared with other ischemic stroke subtypes [10]. However, there are some disputed results on the relationship between $\mathrm{WMH}$ and ICH. It was reported that the presence of WMH is an independent risk factor for warfarin-related ICH and ICH after thrombolytic treatment for acute ischemic stroke [11-13]. Severe WMH are associated with larger ICH volumes and hematoma growth [14]. Increased WMH is an independent predictor of worse functional outcomes in patients after spontaneous ICH [15]. But Dr. Sykora showed that WMH were not associated with initial hematoma volume, hematoma growth, or intraventricular extension. MH were associated with poor outcome independently [16]. Recently, Dr. Boulouis also indicated that the volume of $\mathrm{WMH}$ was not related to either hematoma volume or expansion in ICH [6]. Here, we test our hypothesis that the severity and progression of WMH are related to hematoma volume and absorption in retrospective study.

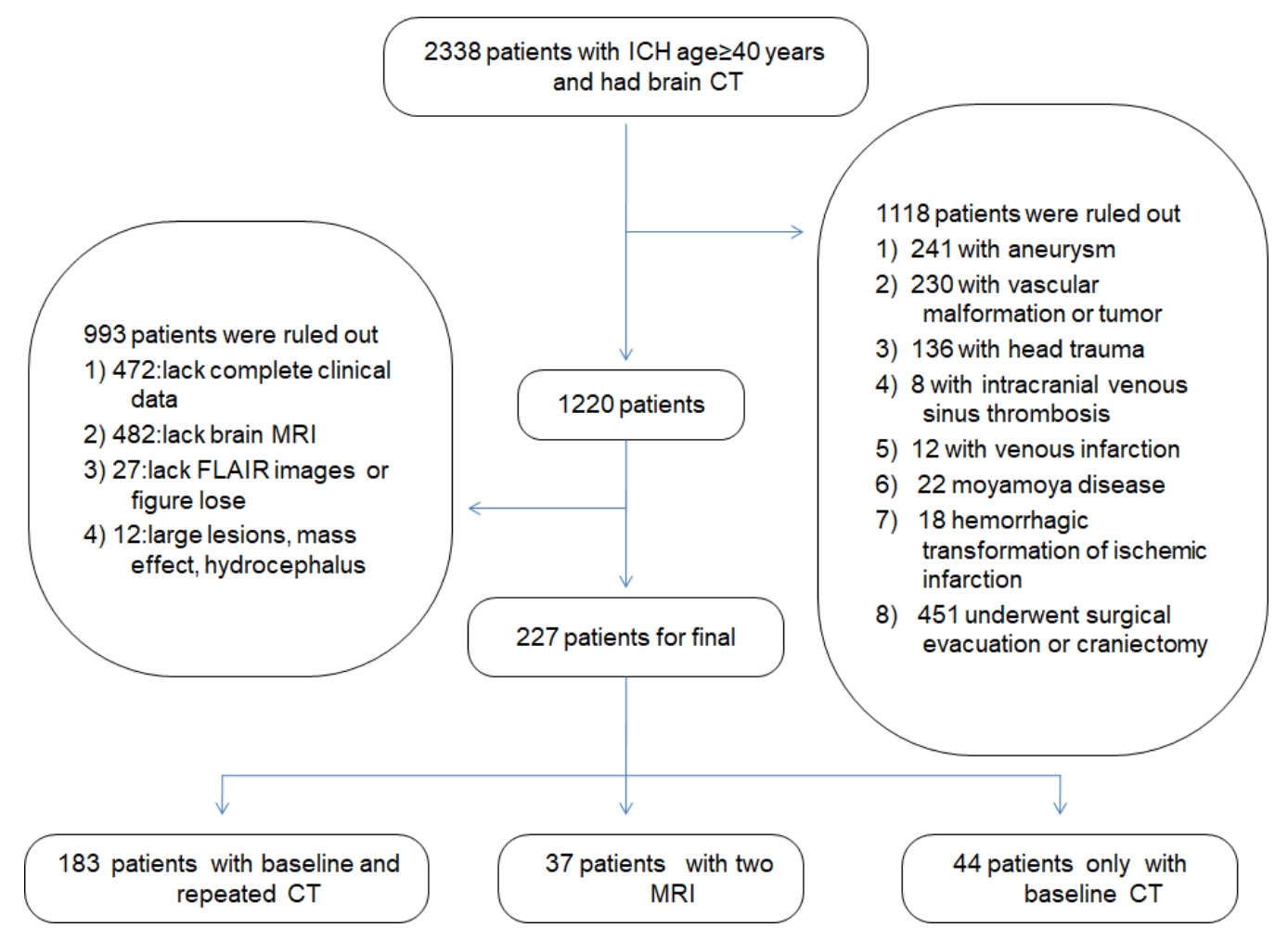

Figure 1. Diagrammatic sketch of the screening process.

\section{MATERIALS AND METHODS}

\section{Study population}

A total of 2338 patients with ICH were studied retrospectively from June 1, 2012, to June 1, 2017, using data from the Drum Tower Hospital imaging center of
Nanjing University Medical School. Patients $\geq 40$ years of age were recruited if they had undergone brain computed tomography (CT) within 12 hours of onset of $\mathrm{ICH}$ symptoms and received fluid attenuated inversion recovery (FLAIR) and magnetic resonance imaging (MRI) after ICH, and if their baseline clinical and demographic information, including demographic 
characteristics, medical history, physical examination results and laboratory examination findings, were available. The exclusion criteria included underlying aneurysm, vascular malformation or tumor, head trauma, venous infarction, moyamoya disease, hemorrhagic transformation of ischemic infarction, and previous surgical evacuation or craniectomy. Consequently, 2111 patients were ruled out: complete clinical data were lacking for 472 patients, brain MRI data were not available for 482 patients, FLAIR imaging data were unavailable for 27 patients, 241 patients had underlying aneurysm, 230 had vascular malformation or tumor, 136 had head trauma, 8 had intracranial venous sinus thrombosis, 12 had venous infarction, 22 had moyamoya disease, 18 had hemorrhagic transformation of ischemic infarction, and 451 had undergone surgical evacuation or craniectomy, 12 patients were excluded for other reasons. Ultimately, 227 patients, including 44 patients with only baseline CT, 183 patients with baseline and repeated CT and 37 patients with two MRIs were analyzed in this study (Fig. 1). To determine the relation between ICH volume and $\mathrm{WMH}$ progression, we selected a total of 37 patients who had undergone another MRI before ICH (the interval between the two examinations was more than one month). The Ethics Committee of Drum Tower Hospital affiliated to Nanjing University Medical School approved this study. The procedures were conducted according to institutional guidelines.
A

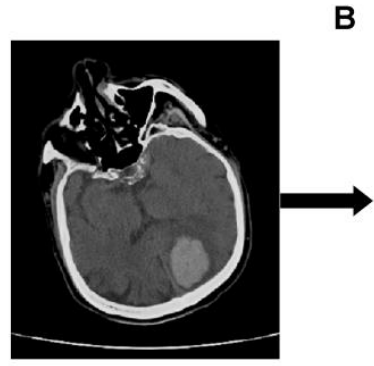

B

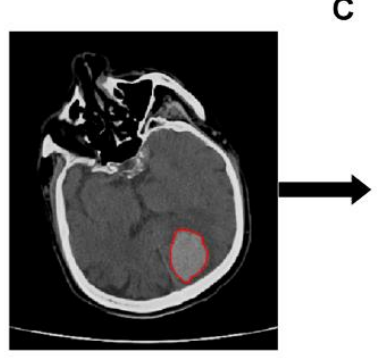

C

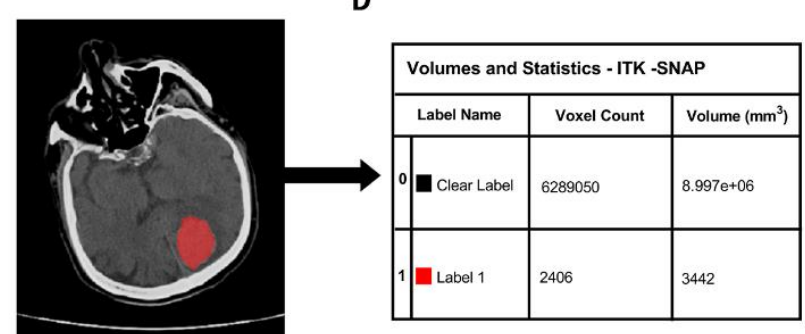

Figure 2. Quantitative steps of ICH volume. (A) Original CT image. (B) The high-density area. (C) A sketch of the high-density area. D) Calculation of ICH volume.

\section{Risk factors}

To assess relevant risk factors at baseline, we examined patient demographic characteristics (age, sex and medical history), history of hypertension, history of diabetes mellitus, presence of coronary heart disease, history of dyslipidemia, and past or present cigarette or alcohol use. Patients underwent a physical examination, systolic and diastolic blood pressure was measured, and laboratory examinations including tests for glucose (random blood glucose RBG, fasting blood glucose FBG, postprandial blood glucose PBG and glycosylated hemoglobin (HbAIc), homocysteine (HCY), total cholesterol (TC), high-density lipoprotein cholesterol (HDL), TG, LDL, apolipoprotein A-I (apoA I), urea nitrogen (BUN), creatinine $(\mathrm{Cr})$, uric acid (UA) and coagulation function were performed.

\section{Radiologic data}

\section{CT acquisition and analysis}

The hematoma volumes of the 227 patients were measured with ITK-SNAP software (University of
Pennsylvania, Philadelphia, USA; www.itksnap.org) at baseline. The baseline scan was limited to 12 hours because most of the hematoma expansion occurred within the following ICH onset after first 24 hours [16]. To determine hematoma absorption, we identified 183 patients who underwent a baseline CT scan within 12 hours and a repeated CT scan within 14 days after ICH onset. We remeasured the repeated ICH volume in 183 patients using ITK-SNAP software. The time window of the repeated CT scan was limited to 14 days after ICH onset because most of the hematoma absorption occurred within 14 days (Fig. 2).

\section{MRI acquisition and analysis}

MRI scanning was performed on a 1.5-T or 3.0-T scanner. The scanning protocol included a whole-brain T1 magnetization-prepared rapid gradient echo sequence, a transversal T2-weighted turbo spin echo sequence, and FLAIR pulse sequences. The timing of the MRI scan was $7.5 \pm 1.5$ days after $\mathrm{ICH}$ onset. 
Table 1. Baseline characteristics classified according to trichotomized Fazekas Score.

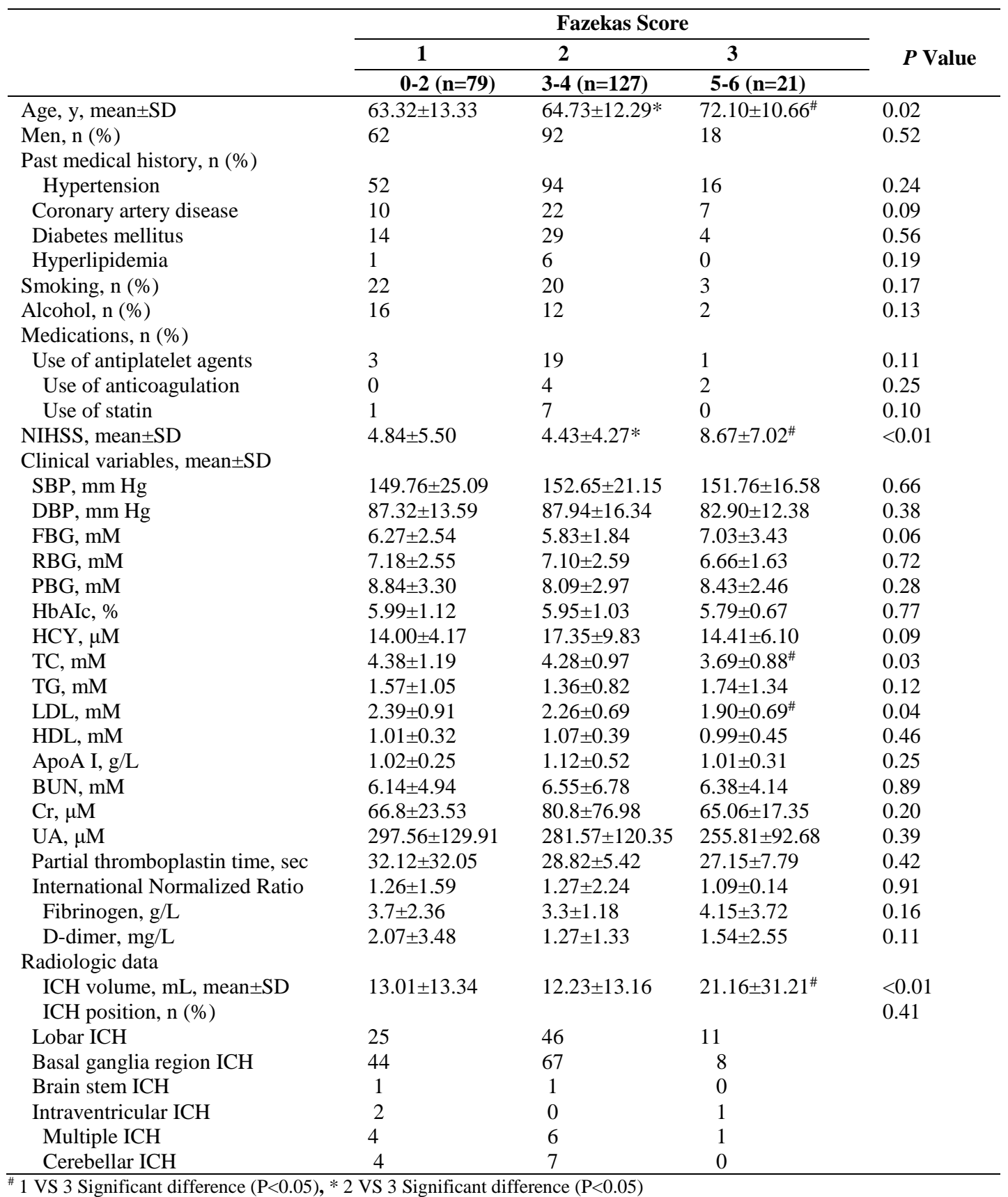

\section{Radiological diagnosis of $\mathrm{WMH}$}

WMHs are hyperintense on T2-weighted or FLAIR sequences but can appear as isointense or hypointense (although less hypointense than CSF) on T1-weighted sequences, depending on the sequence parameters and the severity of the pathological changes [17]. White matter lesions, characterized by bilateral and mostly symmetrical hyperintensities on T2-weighted MRI, are common in older individuals.

\section{Fazekas scale score}

The degree of WMH severity was rated visually on axial FLAIR images using the modified Fazekas scale [18-19], which is the most widely used and validated system for describing WMH severity. This scale divides WMHs into periventricular and deep sites, and periventricular WMHs were graded according to the following patterns: $0=$ absent; 1 = caps or pencil-thin lining; 2 = smooth halo; and $3=$ irregular periventricular $\mathrm{WMH}$ extending into a 
deep WMH. Deep WMHs were graded according to the following patterns: $0=$ absent; $1=$ punctate foci; $2=$ some confluences of foci; and $3=$ large fused areas. The total score ( 0 to 6$)$ was calculated by adding the scores for periventricular and deep WMHs. Because some hemorrhages obscured the underlying brain parenchyma, we analyzed WMH in the hemisphere without ICH.

Table 2. Univariate Risk Factors for Hemorrhage Volume.

\begin{tabular}{|c|c|c|c|}
\hline & \multicolumn{2}{|c|}{ Hemorrhage Volume } & \multirow[b]{2}{*}{$P$ Value } \\
\hline & $0-50 \%(n=114)$ & $51-100 \%(\mathrm{n}=113)$ & \\
\hline Age, $y$, mean \pm SD & $64.97 \pm 12.73$ & $64.87 \pm 12.73$ & 0.95 \\
\hline Men, n $(\%)$ & 70 & 64 & 0.47 \\
\hline \multicolumn{4}{|l|}{ Past medical history, n (\%) } \\
\hline Hypertension & 82 & 80 & 0.85 \\
\hline Coronary artery disease & 12 & 19 & 0.17 \\
\hline Diabetes mellitus & 23 & 20 & 0.63 \\
\hline Hyperlipidemia & 3 & 3 & 0.99 \\
\hline Smoking, n (\%) & 21 & 17 & 0.50 \\
\hline Alcohol, n (\%) & 14 & 10 & 0.40 \\
\hline \multicolumn{4}{|l|}{ Medications, $\mathrm{n}(\%)$} \\
\hline Use of antiplatelet agents & 11 & 8 & 0.49 \\
\hline Use of anticoagulation & 1 & 3 & 0.31 \\
\hline Use of statin & 4 & 3 & 0.51 \\
\hline NIHSS, mean \pm SD & $3.92 \pm 4.00$ & $6.01 \pm 5.91$ & $<0.01 *$ \\
\hline \multicolumn{4}{|l|}{ Clinical variables, mean $\pm \mathrm{SD}$} \\
\hline $\mathrm{SBP}, \mathrm{mm} \mathrm{Hg}$ & $151.47 \pm 24.65$ & $151.65 \pm 19.53$ & 0.95 \\
\hline $\mathrm{DBP}, \mathrm{mm} \mathrm{Hg}$ & $86.68 \pm 15.88$ & $87.87 \pm 14.62$ & 0.56 \\
\hline $\mathrm{FBG}, \mathrm{mM}$ & $5.91 \pm 2.15$ & $6.27 \pm 2.44$ & 0.24 \\
\hline $\mathrm{RBG}, \mathrm{mM}$ & $7.30 \pm 2.72$ & $6.86 \pm 2.23$ & 0.22 \\
\hline PBG, mM & $8.54 \pm 3.06$ & $8.23 \pm 3.06$ & 0.49 \\
\hline HbAIc, $\%$ & $6.03 \pm 1.12$ & $5.87 \pm 0.93$ & 0.32 \\
\hline $\mathrm{HCY}, \mu \mathrm{M}$ & $15.81 \pm 9.38$ & $15.93 \pm 6.41$ & 0.93 \\
\hline $\mathrm{TC}, \mathrm{mM}$ & $4.21 \pm 1.07$ & $4.29 \pm 1.04$ & 0.56 \\
\hline $\mathrm{TG}, \mathrm{mM}$ & $1.47 \pm 0.91$ & $1.46 \pm 1.02$ & 0.87 \\
\hline LDL, mM & $2.25 \pm 0.751$ & $2.28 \pm 0.81$ & 0.83 \\
\hline HDL, mM & $1.06 \pm 0.31$ & $1.02 \pm 0.42$ & 0.39 \\
\hline ApoA I, g/L & $1.12 \pm 0.52$ & $1.02 \pm 0.31$ & 0.08 \\
\hline BUN, mM & $6.03 \pm 6.13$ & $6.75 \pm 5.79$ & 0.37 \\
\hline $\mathrm{Cr}, \mu \mathrm{M}$ & $72.04 \pm 33.02$ & $76.92 \pm 78.09$ & 0.54 \\
\hline $\mathrm{UA}, \mu \mathrm{M}$ & $285.7 \pm 108.19$ & $283.72 \pm 148.16$ & 0.91 \\
\hline Partial thromboplastin time, sec & $28.44 \pm 7.04$ & $31.22 \pm 26.89$ & 0.30 \\
\hline International Normalized Ratio & $1.40 \pm 2.69$ & $1.09 \pm 0.34$ & 0.23 \\
\hline Fibrinogen, g/l & $3.56 \pm 2.56$ & $3.49 \pm 1.20$ & 0.79 \\
\hline D-dimer, mg/l & $1.06 \pm 1.19$ & $2.12 \pm 3.19$ & $<0.01 *$ \\
\hline \multicolumn{4}{|l|}{ Radiologic data } \\
\hline $\mathrm{WMH}$ volume, $\mathrm{mm}^{3}$, mean $\pm \mathrm{SD}$ & $13345.29 \pm 10768.43$ & $16510.27 \pm 9888.57$ & $0.02 *$ \\
\hline ICH position, $\mathrm{n}(\%)$ & & & $0.01 *$ \\
\hline Lobar ICH & 29 & 53 & \\
\hline Basal ganglia region $\mathrm{ICH}$ & 71 & 48 & \\
\hline Brain stem ICH & 2 & 0 & \\
\hline Intraventricular ICH & 1 & 2 & \\
\hline Multiple ICH & 5 & 6 & \\
\hline Cerebellar ICH & 6 & 4 & \\
\hline
\end{tabular}

\section{Quantitative analysis of WMH volume}

WMH volumes were quantified using the software programs MRICRON (University of Nottingham School of Psychology, Nottingham, UK; www.mricro.com) and
ITK-SNAP (Fig. 3). All scans were checked by visual inspection. First, the MRICRON software was used to extract the effective WMH area, and then, the ITK-SNAP software was used to calculate the WMH volume. Because WMH quantification was affected by hematoma 
and perihematomal edema, we adopted certain expedients to reduce errors: we excluded cases with very large lesions, mass effects, or hydrocephalus; the time of MRI scan was $7.5 \pm 1.5$ days after ICH onset to minimize the influence of edema on quantitative WMH measurements.

\section{Statistical analysis}

Mean (standard deviation) or median (interquartile range) values were adopted to determine whether continuous variables had a normal distribution. Frequencies were used to describe categorical variables. Patients were dichotomized according to baseline ICH volume as measured on CT. The proportion of ICH volume absorption was calculated by subtracting the baseline $\mathrm{ICH}$ volume from the follow-up ICH volume on CT scans and dividing this value by the baseline ICH volume. The proportion of ICH absorption was dichotomized according to the repeated ICH volume as measured on CT. To determine the relation between ICH and WMH progression, we defined non-progression as a white matter volume on the second measurement less than or equal to that of the first measurement, and we defined progression as a white matter volume on the second measurement greater than that of the first measurement. We used Bonferroni correction to evaluate differences between the two groups. We used Fisher's exact test to compare dichotomous variables between groups and used the Wilcoxon rank-sum test for comparisons of continuous and ordinal variables. Variables with a $\mathrm{P}<0.1$ based on univariate regression analyses were included in the multivariate logistic regression models. Statistical significance was set at $\mathrm{P}<0.05$.
A

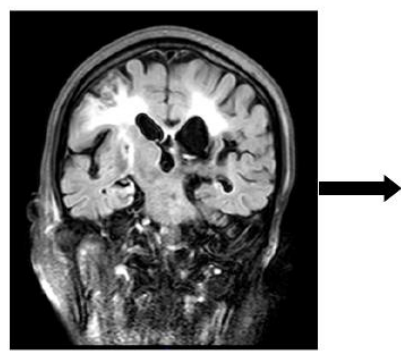

B

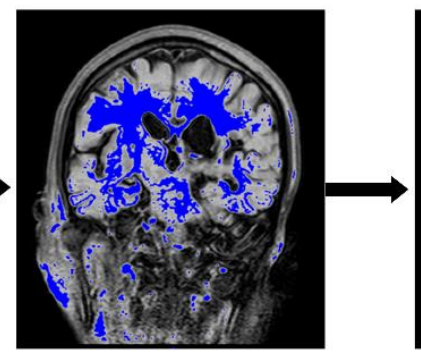

C

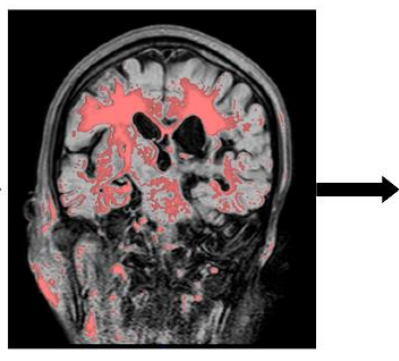

D

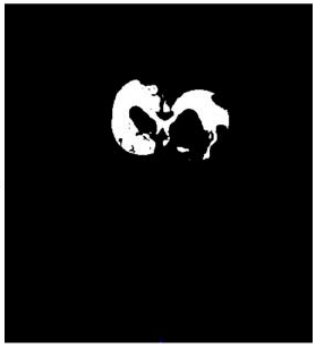

Figure 3. Quantitative steps of WMH volume. (A) Original FLAIR image. (B) The high signal area. C) A sketch of the effective WMH area. (D) Extraction of the effective WMH area.

\section{RESULTS}

\section{Baseline characteristics classified according to trichotomized Fazekas scale scores}

According to the Fazekas scale score, the 227 patients were divided into three groups: Group 1 (score of 0-2), Group 2 (score of 3-4), and Group 3 (score of 5-6). As shown in Table 1, Groups 1 and 2werecompared to Group 3. Patients with higher WMH scores were older and had higher NIHSS scores. When Group 1 was compared to Group 3, patients with higher WMH scores had lower TC levels, lower LDL cholesterol levels, and larger ICH volume $(\mathrm{P}<0.05)$. However, when Group 1 was compared to Group 2 or when Group 2 was compared to Group 3, no significant differences were observed.

\section{Univariate and multivariate logistic regression analyses of risk factors related to ICH volume}

As shown in Table 2, the hemorrhage volume was correlated with the NIHSS score, apoA I level, D-dimer level, WMH volume and ICH site $(\mathrm{P}<0.05)$. Lobar ICH had a greater ICH volume, but in cases of smaller ICH volume, the most common ICH site was the basal ganglia region. Only WMH volume, ICH position and NIHSS score were independently associated with ICH volume based on the multivariate regression results, as shown in Table 3.

Table 3. Multivariate logistic regression analysis of risk factors for ICH volume.

\begin{tabular}{llll}
\hline & Odds Ratio & 95\% CI & $\boldsymbol{P}$ Value \\
\hline NIHSS & 1.08 & $1.01-1.15$ & $0.03^{*}$ \\
D-Dimer & 1.23 & $0.99-1.53$ & 0.06 \\
WMH volume & 1.00 & $1.00-1.00$ & $0.049^{*}$ \\
ApoA I & 0.49 & $0.20-1.80$ & 0.37 \\
ICH position & 1.44 & $1.09-1.91$ & $0.01^{*}$ \\
\hline * Significant difference $(\mathrm{P}<0.05)$ & &
\end{tabular}


Table 4. Analysis of relationship of hematoma volume and WMH progression.

\begin{tabular}{|c|c|c|c|}
\hline & \multicolumn{3}{|c|}{ WMH progress } \\
\hline & No $(n=17)$ & Yes $(n=20)$ & $P$ Value \\
\hline Age, $y$, mean \pm SD & $59.24 \pm 19.43$ & $57.90 \pm 15.60$ & 0.82 \\
\hline Men, n $(\%)$ & 12 & 14 & 0.97 \\
\hline \multicolumn{4}{|l|}{ Past medical history, n (\%) } \\
\hline Hypertension & 7 & 12 & 0.25 \\
\hline Coronary artery disease & 0 & 1 & 0.26 \\
\hline Diabetes mellitus & 3 & 3 & 0.83 \\
\hline Hyperlipidemia & 1 & 0 & 0.21 \\
\hline Smoking, n (\%) & 1 & 4 & 0.19 \\
\hline Alcohol, n (\%) & 1 & 5 & 0.10 \\
\hline \multicolumn{4}{|l|}{ Medications, n (\%) } \\
\hline Use of antiplatelet agents & 0 & 1 & 0.26 \\
\hline Use of anticoagulation & 0 & 0 & - \\
\hline Use of statin & 1 & 0 & 0.21 \\
\hline \multicolumn{4}{|l|}{ Clinical variables, mean \pm SD } \\
\hline $\mathrm{SBP}, \mathrm{mm} \mathrm{Hg}$ & $136.65 \pm 21.92$ & $145.20 \pm 19.17$ & 0.21 \\
\hline $\mathrm{DBP}, \mathrm{mm} \mathrm{Hg}$ & $77.35 \pm 12.61$ & $83.75 \pm 10.56$ & 0.10 \\
\hline $\mathrm{FBG}, \mathrm{mM}$ & $5.61 \pm 1.75$ & $5.48 \pm 1.72$ & 0.82 \\
\hline $\mathrm{RBG}, \mathrm{mM}$ & $7.32 \pm 4.11$ & $6.99 \pm 2.70$ & 0.80 \\
\hline $\mathrm{PBG}, \mathrm{mM}$ & $8.43 \pm 4.09$ & $7.90 \pm 2.03$ & 0.67 \\
\hline HbAIc, \% & $6.07 \pm 0.67$ & $6.02 \pm 1.29$ & 0.93 \\
\hline $\mathrm{TC}, \mathrm{mM}$ & $4.02 \pm 1.29$ & $3.79 \pm 0.69$ & 0.57 \\
\hline $\mathrm{TG}, \mathrm{mM}$ & $1.13 \pm 0.44$ & $1.37 \pm 0.83$ & 0.38 \\
\hline LDL, mM & $2.15 \pm 0.95$ & $1.98 \pm 0.52$ & 0.58 \\
\hline HDL, mM & $1.00 \pm 0.28$ & $0.93 \pm 0.27$ & 0.57 \\
\hline ApoA I, g/L & $1.06 \pm 0.25$ & $0.98 \pm 0.27$ & 0.41 \\
\hline BUN, mM & $6.54 \pm 3.41$ & $5.56 \pm 1.50$ & 0.34 \\
\hline $\mathrm{Cr}, \mu \mathrm{M}$ & $87.17 \pm 31.23$ & $69.29 \pm 25.03$ & 0.12 \\
\hline $\mathrm{UA}, \mu \mathrm{M}$ & $319.00 \pm 112.34$ & $297.71 \pm 118.94$ & 0.65 \\
\hline Partial thromboplastin time, sec & $32.21 \pm 5.19$ & $32.73 \pm 11.95$ & 0.89 \\
\hline International Normalized Ratio & $1.10 \pm 0.07$ & $1.14 \pm 0.28$ & 0.65 \\
\hline Fibrinogen, g/L & $2.85 \pm 1.10$ & $3.36 \pm 1.11$ & 0.26 \\
\hline D-dimer, mg/L & $1.39 \pm 1.21$ & $1.20 \pm 1.01$ & 0.70 \\
\hline \multicolumn{4}{|l|}{ Radiologic data } \\
\hline $\mathrm{ICH}$ volume, $\mathrm{ml}$, mean $\pm \mathrm{SD}$ & $7.12 \pm 9.19$ & $13.59 \pm 10.25$ & $0.049^{*}$ \\
\hline
\end{tabular}

* Significant difference $(\mathrm{P}<0.05)$

\section{Analysis of the relationship between hematoma volume and WMH progression}

As shown in Table 4, of all 227 patients, thirty-seven patients who had received two MRIs before ICH onset were analyzed (the interval between the two examinations was more than one month). In this subset of patients, WMH progression was correlated with ICH volume $(\mathrm{P}<0.05)$. However, a trend was observed only for the association between WMH progression and hematoma volume because of the low sample size.

Univariate and multivariate logistic regression analyses of risk factors related to the proportion of hemorrhage absorption

Hemorrhage absorption was correlated with ICH volume, history of hypertension and postprandial blood glucose
(PBG). However, no trend of an association between WMH volume and proportion of hematoma absorption was observed (Table 5). After adjusting for other confounding variables that could influence ICH absorption, only high ICH volume was independently associated with a high proportion of ICH absorption based on multivariate regression (Table 6).

\section{DISCUSSION}

The main findings of this study were as follows: 1) there was an independent association between the severity of $\mathrm{WMH}$ and the ICH volume in this cohort of patients, according to quantitative WMH measurements and Fazekas scale scores; 2) WMH progression was correlated with increased ICH volume, suggesting that white matter damage might predict ICH volume. 
Table 5. Univariate analysis of hematoma absorption proportion.

\begin{tabular}{|c|c|c|c|}
\hline & \multicolumn{2}{|c|}{ Hemorrhage Absorption Proportion } & \multirow[b]{2}{*}{$P$ Value } \\
\hline & $0-50 \%(n=48)$ & $51-100 \%(n=63)$ & \\
\hline Age, $y$, mean \pm SD & $64.35 \pm 12.65$ & $65.47 \pm 12.42$ & 0.56 \\
\hline Men, n $(\%)$ & 48 & 63 & 0.11 \\
\hline \multicolumn{4}{|l|}{ Past medical history, $\mathrm{n}(\%)$} \\
\hline Hypertension & 48 & 91 & $0.03 *$ \\
\hline Coronary artery disease & 10 & 12 & 0.64 \\
\hline Diabetes mellitus & 11 & 24 & 0.34 \\
\hline Hyperlipidemia & 0 & 3 & 0.28 \\
\hline Smoking, n (\%) & 11 & 21 & 0.56 \\
\hline Alcohol, n (\%) & 8 & 10 & 0.80 \\
\hline \multicolumn{4}{|l|}{ Medications, n (\%) } \\
\hline Use of antiplatelet agents & 4 & 12 & 0.29 \\
\hline Use of anticoagulation & 1 & 3 & 0.49 \\
\hline Use of statin & 1 & 4 & 0.65 \\
\hline NIHSS, mean \pm SD & $5.44 \pm 6.00$ & $4.58 \pm 4.50$ & 0.28 \\
\hline \multicolumn{4}{|l|}{ Clinical variables, mean \pm SD } \\
\hline $\mathrm{SBP}, \mathrm{mm} \mathrm{Hg}$ & $149.73 \pm 26.85$ & $153.32 \pm 19.69$ & 0.30 \\
\hline $\mathrm{DBP}, \mathrm{mm} \mathrm{Hg}$ & $87.75 \pm 15.76$ & $87.88 \pm 14.99$ & 0.95 \\
\hline $\mathrm{FBG}, \mathrm{mM}$ & $5.94 \pm 2.26$ & $5.88 \pm 1.89$ & 0.84 \\
\hline $\mathrm{RBG}, \mathrm{mM}$ & $6.95 \pm 2.45$ & $7.31 \pm 2.65$ & 0.40 \\
\hline $\mathrm{PBG}, \mathrm{mM}$ & $7.72 \pm 2.54$ & $8.79 \pm 3.34$ & $0.04 *$ \\
\hline HbAIc, $\%$ & $5.87 \pm 0.92$ & $6.06 \pm 1.15$ & 0.33 \\
\hline $\mathrm{HCY}, \mu \mathrm{M}$ & $14.74 \pm 7.54$ & $17.45 \pm 8.94$ & 0.14 \\
\hline $\mathrm{TC}, \mathrm{mM}$ & $4.25 \pm 1.06$ & $4.28 \pm 0.99$ & 0.82 \\
\hline $\mathrm{TG}, \mathrm{mM}$ & $1.38 \pm 1.00$ & $1.39 \pm 0.78$ & 0.97 \\
\hline LDL, mM & $2.24 \pm 0.83$ & $2.31 \pm 0.71$ & 0.54 \\
\hline HDL, mM & $1.04 \pm 0.38$ & $1.07 \pm 0.39$ & 0.69 \\
\hline ApoA I, g/L & $1.04 \pm 0.29$ & $1.11 \pm 0.53$ & 0.32 \\
\hline BUN, mM & $5.74 \pm 2.73$ & $6.40 \pm 7.07$ & 0.45 \\
\hline $\mathrm{Cr}, \mu \mathrm{M}$ & $66.81 \pm 27.10$ & $79.78 \pm 80.87$ & 0.19 \\
\hline $\mathrm{UA}, \mu \mathrm{M}$ & $276.62 \pm 94.89$ & $272.38 \pm 123.87$ & 0.81 \\
\hline Partial thromboplastin time, sec & $29.33 \pm 5.57$ & $27.99 \pm 5.49$ & 0.12 \\
\hline International Normalized Ratio & $1.06 \pm 0.20$ & $1.29 \pm 2.41$ & 0.42 \\
\hline Fibrinogen, g/l & $3.49 \pm 1.14$ & $3.57 \pm 1.87$ & 0.78 \\
\hline D-dimer, mg/l & $1.91 \pm 2.99$ & $1.41 \pm 2.30$ & 0.26 \\
\hline \multicolumn{4}{|l|}{ Radiologic data } \\
\hline $\mathrm{WMH}$ volume, $\mathrm{mm} 3$, mean $\pm \mathrm{SD}$ & $15344.49 \pm 10501.54$ & $14939.61 .82 \pm 9674.11$ & 0.79 \\
\hline Fazakse score, mean \pm SD & $2.06 \pm 1.12$ & $2.09 \pm 0.97$ & 0.83 \\
\hline $\mathrm{ICH}$ volume, $\mathrm{mL}$, mean $\pm \mathrm{SD}$ & $16045.12 \pm 14055.45$ & $11720.40 \pm 12750.35$ & $0.03 *$ \\
\hline ICH position, n (\%) & & & 0.16 \\
\hline Lobar ICH & 26 & 34 & \\
\hline Basal ganglia region ICH & 37 & 65 & \\
\hline Brain stem ICH & 1 & 1 & \\
\hline Intraventricular ICH & -- & -- & \\
\hline Multiple ICH & 1 & 7 & \\
\hline Cerebellar ICH & 6 & 3 & \\
\hline
\end{tabular}

* Significant difference $(\mathrm{P}<0.05)$

WMH is a neuroimaging finding of white matter changes and is thought to be a result of ischemic injury and demyelination [20-21]. WMHs are related to ischemic stroke [21-22], ICH, global functional decline [23], and dementia [24]. WMH has been considered to reflect damage to small vessels in periventricular and subcortical areas, and autopsy studies have shown that the pathogenesis of WMH includes myelin pallor, dilatation of perivascular spaces and tissue myelin rarefaction [2526]. $\mathrm{WMH}$ is associated with certain vascular risk factors, such as age, hypertension, diabetes mellitus, dyslipidemia, and smoking [26-28]. As shown in our study, the group with the highest Fazekas scale scores had significantly higher age, NIHSS score, and ICH volume but lower TC and LDL cholesterol levels than the group with the lowest Fazekas scale scores $(\mathrm{P}<0.05)$. The more serious the WMH was, the more significant these differences were. The NIHSS is a 15 -item neurological function scale used in patients with acute stroke. A higher NIHSS score reflects a worse patient condition. Higher 
Fazekas scale scores suggest a greater degree of white matter and brain damage, and the Fazekas scale score has a positive correlation with the NIHSS score.

$\mathrm{ICH}$ is the second most common subtype of stroke and is a critical disease that usually leads to severe disability or death. Spontaneous ICH has been attributed to the rupture of arteries previously damaged by chronic hypertension. Pathologic changes in ICH include microaneurysms, fibrinoid degeneration and lipohyalinosis. Compared to ischemic stroke, ICH has higher mortality and more severe disability [29]. Therefore, it is important to study the pathophysiology, risk factors, and prognostic factors of $\mathrm{ICH}$. ICH and $\mathrm{WMH}$ share several risk factors in common (hypertension, cerebrovascular disease) and may share a common underlying pathological mechanism involving microangiopathy [15]. As a surrogate for cerebral microangiopathy, WMH has been previously associated with increased risk of spontaneous and medicationinduced intraparenchymal hemorrhage putatively via changes in vessel wall integrity, disruption of the brainblood barrier, and loss of tissue density [8, 30, 31]. The extent of this pathology may contribute to more severe hematomas, more frequent hemorrhage growth, and intraventricular extension. In this study, we dichotomized patients by hemorrhage volume, and we found a significant association between the severity of $\mathrm{WMH}$ and hemorrhage volume, which is consistent with the results of Lou [14]. Moreover, in the subgroup in which the relationship between WMH progression and ICH volume was analyzed, we found a positive relationship between the progression of white matter changes and larger ICH volumes. These common pathophysiologic features between WMH and ICH and the impact of WMHs on brain density provide mechanistic links accounting for our findings, whereby hypertension or cerebral amyloid angiopathy (CAA)may play a role in the progression of WMH in association with ICH.

NIHSS scores indicated the degree of disease severity and were closely related to the ICH volume. Higher NIHSS scores were associated with larger ICH volumes. As shown in this study. The ICH site was also associated with ICH volume based on multivariate analysis. It is commonly noted that cerebral hemorrhage occurs most frequently in the lobar and basal ganglia regions. In this study, lobar hemorrhage was associated with greater ICH volume. The pathophysiologic basis for our findings and its potential relation to $\mathrm{WMH}$ that frequently accompanies CAA require further investigation.

Known poor prognostic factors of ICH include large hematoma volume, hematoma expansion and absorption, intraventricular hemorrhage, infra-tentorial location, older age, and anticoagulation treatment [5]. With regard to acute brain bleeding, a large case-control study showed that extensive white matter lesions were associated with lower Glasgow Coma Scale scores and higher mortality [5]. However, our study did not find a clear relationship between the severity of WMH and ICH absorption, as ICH absorption was related to only the initial ICH volume. Further research is needed to explore the relationship between the burden of WMH and ICH absorption.

Table 6. Multivariate Logistic Regression Analysis of Risk Factors for Hematoma Absorption Proportion.

\begin{tabular}{llll}
\hline & Odds Ratio & $\mathbf{9 5 \%}$ CI & $\boldsymbol{P}$ Value \\
\hline Hypertension & 0.56 & $0.24-1.33$ & 0.19 \\
ICH volume & 1.00 & $1.00-1.00$ & $0.02 *$ \\
PBG & 1.12 & $0.99-1.28$ & 0.08 \\
\hline
\end{tabular}

* Significant difference $(\mathrm{P}<0.05)$

\section{Limitations}

The limitations of our study are as follows. First, this was a single-center, retrospective cohort study design, which should not be extensively relied upon in clinical practice. Future prospective studies should address these limitations. Second, hematoma growth was examined in a small subset of 37 patients. These results, therefore, should be considered preliminary and hypothesisgenerating. Further prospective investigations in larger cohorts are needed to confirm the results. Finally, although some measures have been taken to reduce error, the calculated white matter volume was not accurate because the WMH quantification was affected by many factors. Recently, it has been shown that diffusion tensor imaging (DTI) is useful for detecting microstructural abnormalities in white matter. Thus, DTI is expected to supersede conventional methods of WMH measurement in the future [32].

\section{Conclusions}

In conclusion, it was found that the severity and progression of WMH were correlated with ICH volume in patients with ICH. Our findings suggest that WMH may provide important prognostic information about patients with ICH and provide implications for the prevention and treatment of WMH formation or progression. To reduce the occurrence of $\mathrm{ICH}$ and improve the functional outcomes of patients after ICH, WMH should be addressed at the early stages of WMH development in clinical practice. Further studies are needed to elucidate the pathophysiologic link between WMH and ICH and to confirm our findings. 


\section{Acknowledgements}

This research was supported by the National Natural Science Foundation of China (81230026, 81630028, 81771157), the Science and Technology Department of Jiangsu Province (BE2016610), Jiangsu Province Key Medical Discipline (ZDXKA2016020) and Nanjing Medical Science and technique Development Foundation (QRX17032).

\section{References}

[1] Qureshi AI, Mendelow AD, Hanley DF (2009). Intracerebral haemorrhage. Lancet, 373:1632-1644.

[2] Zuo S, Pan P, Li Q, Chen Y, Feng H (2017). White matter injury and recovery after hypertensive intracerebral hemorrhage. Biomed Res Int. 2017:6138424.

[3] Feigin VL, Lawes CM, Bennett DA, Barker-Collo SL, Parag V (2009). Worldwide stroke incidence and early case fatality reported in 56 population-based studies: A systematic review. Lancet Neurol, 8:355-369.

[4] Sacco S, Marini C, Toni D, Olivieri L, Carolei A (2009). Incidence and 10-year survival of intracerebral hemorrhage in a population-based registry. Stroke, 40:394-399.

[5] An SJ, Kim TJ, Yoon BW (2017). Epidemiology, risk factors, and clinical features of intracerebral hemorrhage: An update. J Stroke, 19:3-10.

[6] Boulouis G, van Etten ES, Charidimou A, Auriel E, Morotti A, Pasi M, et al (2016). Association of key magnetic resonance imaging markers of cerebral small vessel disease with hematoma volume and expansion in patients with lobar and deep intracerebral hemorrhage. JAMA Neurol, 73:1440-1447.

[7] Wardlaw JM, Smith EE, Biessels GJ, Cordonnier C, Fazekas F, Frayne R, et al (2013). Neuroimaging standards for research into small vessel disease and its contribution to ageing and neurodegeneration. Lancet Neurol, 12:822-838.

[8] Pantoni L, Garcia JH (1997). Pathogenesis of leukoaraiosis: A review. Stroke, 28:652-659.

[9] Lee SH, Kim BJ, Ryu WS, Kim CK, Kim N, Park BJ (2010). White matter lesions and poor outcome after intracerebral hemorrhage: A nationwide cohort study. Neurology, 74:1502-1510.

[10] Rost NS, Rahman RM, Biffi A, Smith EE, Kanakis A, Fitzpatrick K, et al (2010). White matter hyperintensity volume is increased in small vessel stroke subtypes. Neurology, 75:1670-1677.

[11] Folsom AR, Yatsuya H, Mosley TH, Jr., Psaty BM, Longstreth WT, Jr (2012). Risk of intraparenchymal hemorrhage with magnetic resonance imaging-defined leukoaraiosis and brain infarcts. Ann Neurol, 71:552559.

[12] Curtze S, Haapaniemi E, Melkas S, Mustanoja S, Putaala J, Sairanen T, et al (2015). White matter lesions double the risk of post-thrombolytic intracerebral hemorrhage. Stroke, 46:2149-2155.
[13] Smith EE, Rosand J, Knudsen KA, Hylek EM, Greenberg SM (2002). Leukoaraiosis is associated with warfarin-related hemorrhage following ischemic stroke. Neurology, 59:193-197.

[14] Lou M, Al-Hazzani A, Goddeau RP, Jr., Novak V, Selim M (2010). Relationship between white-matter hyperintensities and hematoma volume and growth in patients with intracerebral hemorrhage. Stroke, 41:3440.

[15] Caprio FZ, Maas MB, Rosenberg NF, Kosteva AR, Bernstein RA, Alberts MJ, et al (2013). Leukoaraiosis on magnetic resonance imaging correlates with worse outcomes after spontaneous intracerebral hemorrhage. Stroke, 44:642-646.

[16] Sykora M, Herweh C, Steiner T (2017). The association between leukoaraiosis and poor outcome in intracerebral hemorrhage is not mediated by hematoma growth. J Stroke Cerebrovasc Dis, 26:1328-1333.

[17] Brott T, Broderick J, Kothari R, Barsan W, Tomsick T, Sauerbeck L, et al (1997). Early hemorrhage growth in patients with intracerebral hemorrhage. Stroke, 28:1-5.

[18] Fazekas F, Chawluk JB, Alavi A, Hurtig HI, Zimmerman RA (1987). MR signal abnormalities at $1.5 \mathrm{~T}$ in alzheimer's dementia and normal aging. AJR Am J Roentgenol, 149:351-356.

[19] Boutet C, Rouffiange-Leclair L, Schneider F, Camdessanche JP, Antoine JC, Barral FG (2016). Visual assessment of age-related white matter hyperintensities using flair images at $3 \mathrm{~T}$ : Inter- and intra-rater agreement. Neurodegener Dis, 16:279-283.

[20] Bivard A, Cheng X, Lin LT, Levi C, Spratt N, Kleinig T, et al (2016). Global white matter hypoperfusion on ct predicts larger infarcts and hemorrhagic transformation after acute ischemia. CNS Neurosci Ther, 22:238-243.

[21] Ay H, Arsava EM, Rosand J, Furie KL, Singhal AB, Schaefer PW, et al (2008). Severity of leukoaraiosis and susceptibility to infarct growth in acute stroke. Stroke, 39:1409-1413.

[22] Arsava EM, Rahman R, Rosand J, Lu J, Smith EE, Rost NS, et al (2009). Severity of leukoaraiosis correlates with clinical outcome after ischemic stroke. Neurology, 72:1403-1410.

[23] Inzitari D, Pracucci G, Poggesi A, Carlucci G, Barkhof F, Chabriat H, et al (2009). Changes in white matter as determinant of global functional decline in older independent outpatients: Three year follow-up of ladis (leukoaraiosis and disability) study cohort. BMJ, 339: b2477.

[24] Boulouis G, Charidimou A, Auriel E, Haley KE, van Etten ES, Fotiadis P, et al (2016). Intracranial atherosclerosis and cerebral small vessel disease in intracerebral hemorrhage patients. J Neurol Sci, 369:324-329.

[25] Debette S, Markus HS (2010). The clinical importance of white matter hyperintensities on brain magnetic resonance imaging: Systematic review and metaanalysis. BMJ, 341:c3666.

[26] Chowdhury MH, Nagai A, Bokura H, Nakamura E, Kobayashi S, Yamaguchi S (2011). Age-related changes in white matter lesions, hippocampal atrophy, and 
cerebral microbleeds in healthy subjects without major cerebrovascular risk factors. J Stroke Cerebrovasc Dis, 20:302-309.

[27] Luo Y, Li J, Zhang J, Xu Y (2014). Low HDL cholesterol is correlated to the acute ischemic stroke with diabetes mellitus. Lipids Health Dis, 13:171.

[28] Helenius J, Mayasi Y, Henninger N (2017). White matter hyperintensity lesion burden is associated with the infarct volume and 90-day outcome in small subcortical infarcts. Acta Neurol Scand, 135:585-592.

[29] Keep RF, Hua Y, Xi G (2012). Intracerebral haemorrhage: Mechanisms of injury and therapeutic targets. Lancet Neurol, 11:720-731.
[30] Starr JM, Wardlaw J, Ferguson K, MacLullich A, Deary IJ, Marshall I (2003). Increased blood-brain barrier permeability in type ii diabetes demonstrated by gadolinium magnetic resonance imaging. J Neurol Neurosurg Psychiatry, 74:70-76.

[31] Farnaz Farokhian, Chunlan Yang1, Iman Beheshti, Hiroshi Matsuda, Shuicai Wu (2017). Age-Related Gray and White Matter Changes in Normal Adult Brains. Aging Dis, 8(6): 899-909.

[32] Tamura Y, Araki A (2015). Diabetes mellitus and white matter hyperintensity. Geriatr Gerontol Int, 15 Suppl $1: 34-42$. 